\title{
Structural studies on M. smegmatis MutT2, a sanitization enzyme
}

Amandeep Singh ${ }^{1}$, Sheikh Mohammad Arif ${ }^{1}$, Pau Biak Sang ${ }^{2}$, Umesh Varshney ${ }^{2}$, M Vijayan ${ }^{1}$

${ }_{1}^{1}$ Molecular Biophysics Unit, Indian Institute Of Science, Bangalore, India, ${ }^{2}$ Department of Mictobiology and Cell biology, Indian Institute of science, Bangalore, India

E-mail: amandeep@mbu.iisc.ernet.in

Incorporation in DNA of oxidized or damaged nucleotides, such as 8-oxo-dGTP or 5-me-dCTP generated during cellular metabolism or as a response to stress in the host cell, is detrimental to genomic integrity. Apart from a plethora of DNA repair proteins that work directly on the damaged DNA, organisms also posses a set of 'sanitization' enzymes. MutT proteins are among such sanitizing enzymes that function by hydrolyzing such nucleotides, thereby preventing their misincorporation in DNA. Mycobacteria posses a set of 4 MutT homologs, namely, MutT1, MutT2, MutT3 and MutT4. Of these, MutT1 has been biochemically and structurally characterized in the laboratory $[1,2]$. Here we report structural studies of MutT2 from Mycobacterium smegmatis and its complexes with substrates 5me-dCTP, dCTP and CTP and the respective products. The native protein and complexes with products crystallize in space group P21212, with one molecule in the asymmetric unit. However, a majority of substrate complexes crystallize in space group P21, with two molecules in the asymmetric unit.

At a gross level, MutT1 hydrolyses oxidized guanosine nucleotides efficiently, while MutT2 hydrolyses cytosine nucleotides better. Unlike MutT1, which has an additional domain along with the characteristic Nudix domain, MutT2 is a single Nudix domain enzyme. The structure of MutT2 is very similar to single domain homologs: human MutT homolog (MTH1), E. coli MutT and Orf135 proteins. In MutT2, the binding pocket is well formed on the surface and, the substrate and the product bind to the same pocket, unlike in mycobacterial MutT1, in which the product binds at an inter-molecular interface. While the structure of the Nudix domain of MutT1 and MutT2 is very similar, the active and binding site residues exhibit substantial differences. In particular, the loops at the mouth of binding site in MutT2 are longer than in MutT1, a situation similar to that found in EcOrf135 and EcMutT. Another relevant observation is in relation to the depth of the ligand pocket. While the ligand remains closer to surface presumably due to presence of domain 2 in MutT1, the nitrogenous base is positioned relatively deeper in MutT2, a situation similar to that observed in single domain MutT homologs. The work presented here provides insights into the mechanism of action of this enzyme and also into the difference between the molecular basis of the functioning of mycobacterial MutT1 and MutT2.

[1] Sang, P.B. et al. (2013) J. Bacteriol. 195:7, 1552-1560.

[2] Arif, S.M. et al. (2017) Acta Cryst. D. (accepted manuscript)

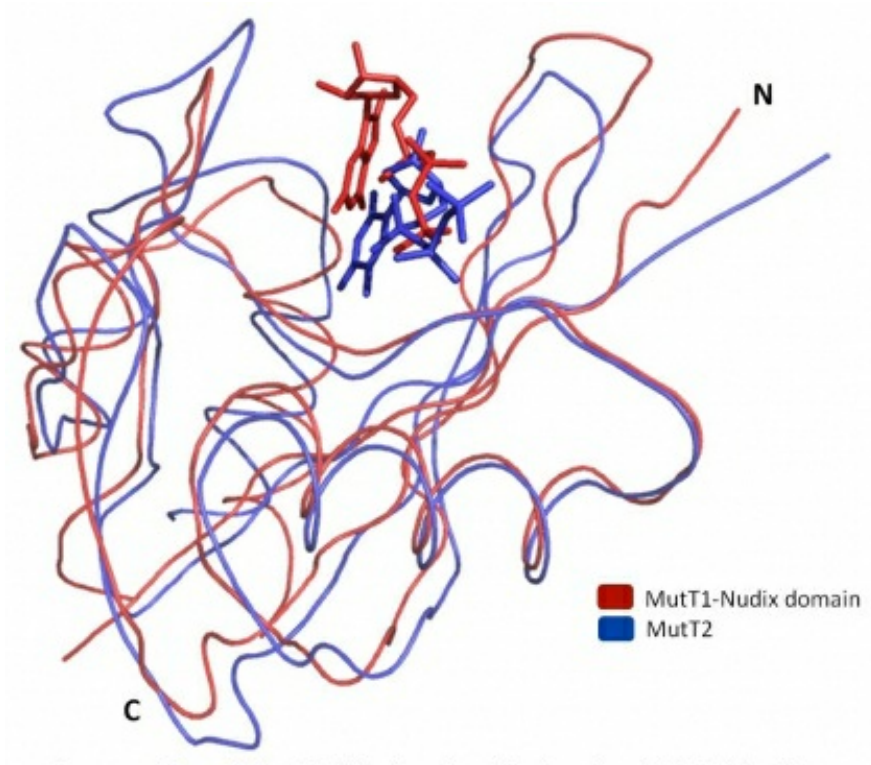

Superposition of MutT2 (blue) and nudix domain of MutT1 (red) Locations of the bound ligands are also indicated.

Keywords: Nudix hydrolase, damaged nucleotides, Mut proteins 